\title{
Complete Cototal Domination Number of a Graph
}

\author{
B. Basavanagoud and S. M. Hosamani* \\ Department of Mathematics, Karnatak University, Dharwad-580 003, India \\ Received 28 May 2011, accepted in final revised form 18 August 2011
}

\begin{abstract}
Let $G=(V, E)$ be a graph. A set $D \subseteq V$ of a graph $G=(V, E)$ is called a total dominating set if the induced subgraph $\langle D\rangle$ has no isolated vertices. The total domination number $\gamma_{t}(G)$ of $G$ is the minimum cardinality of a total dominating set of $G$. A total dominating set $D$ is said to be a complete cototal dominating set if the induced subgraph $\langle V-D\rangle$ has no isolated vertices. The complete cototal domination number $\gamma_{c c}(G)$ of $G$ is the minimum cardinality of a complete cototal dominating set of $G$. In this paper, we initiate the study of complete cototal domination in graphs and present bounds and some exact values for $\gamma_{c c}(G)$. Also its relationship with other domination parameters are established and related two open problems are explored.
\end{abstract}

Keywords: Domination number; Total domination number; Cototal domination number; Complete cototal domination number.

(C) 2011 JSR Publications. ISSN: 2070-0237 (Print); 2070-0245 (Online). All rights reserved. doi:10.3329/jsr.v3i3.7744

J. Sci. Res. 3 (3), 547-555 (2011)

\section{Introduction}

The graphs considered here are simple and without isolated vertices. Let $G=(V, E)$ be a graph with $|V|=p$ and $|E|=q$. A vertex $V$ of a graph $G=(V, E)$ is said to be a pendant vertex if $\operatorname{deg}(v)=1$, while a vertex which is adjacent to a pendant vertex is called the support vertex. The set of pendant vertices of a graph $G$ will be denoted $P(G)$, while the set of all support vertices of $G$ will be denoted by $S(G)$. We denote $\langle D\rangle$ to denote the subgraph induced by the set of vertices $D$ and $N(u)$ and $N[v]$ denote the open and closed neighborhoods of a vertex $v$, respectively. Let $\operatorname{deg}(v)$ be the degree of a vertex $v$ and as usual $\delta(G)$ the minimum degree and $\Delta(G)$ maximum degree. In general, we follow the notation and terminology of Harary [1].

Each vertex of a graph is said to dominate every vertex in its closed neighborhood. A set $D \subseteq V$ is a dominating set if each vertex in $V$ is dominated by some vertex in $D$. The domination number $\gamma(G)$ of $G$ is the minimum cardinality of a dominating set. Many domination parameters have been defined. For comprehensive work on the subject [2]. A dominating set $D \subseteq V$ of a graph $G=(V, E)$ is called a connected dominating set if the induced subgraph $\langle D\rangle$ is connected. The connected domination number $\gamma_{C}(G)$ of $G$ is the minimum cardinality of a connected dominating set of $G$ [3].

\footnotetext{
* Corresponding author: b.basavanagoud@gmail.com
} 
A set $D \subseteq V$ of a graph $G=(V, E)$ is called a total dominating set if the induced subgraph $\langle D\rangle$ has no isolated vertices. The total domination number $\gamma_{t}(G)$ of $G$ is the minimum cardinality of a total dominating set of $G$ [4].

A dominating set $D$ is said to be a cototal dominating set if the induced subgraph $\langle V-D\rangle$ has no isolated vertices. The cototal domination number $\gamma_{C l}(G)$ of $G$ is the minimum cardinality of a cototal dominating set of $G$ [5]. Inspired from the cototal domination, we introduce the new parameter in the field of domination theory of graphs as follows:

A total dominating set $D$ is said to be a complete cototal dominating set if the induced subgraph $\langle V-D\rangle$ has no isolated vertices. The complete cototal domination number $\gamma_{C C}(G)$ of $G$ is the minimum cardinality of a complete cototal dominating set of $G$.

For example, consider a graph $G$ in Fig. 1 . The set $\{1,2,3\}$ is a dominating set as well as cototal dominating set, and set $\{4,5,6\}$ is a total dominating set. However, any complete cototal dominating set must include each pendant vertex $\{1,2,3\}$ and its neighbor $\{4,5,6\}$. Thus $\gamma_{C C}(G)=\{1,2,3,4,5,6\}=|V|=p$.

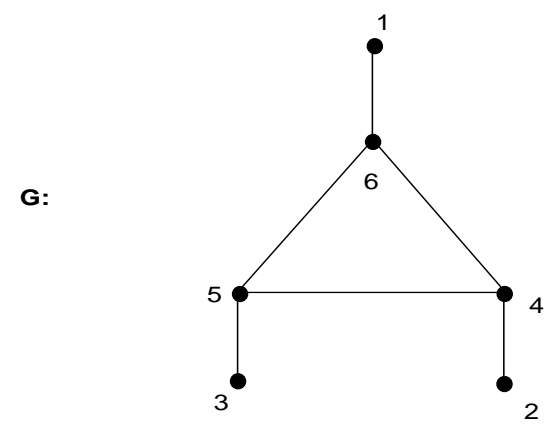

Fig. 1.

\section{Applications}

Many applications of domination in graphs can be extended to complete cototal domination. For example, if we think of each vertex in a dominating set as file server for a computer network, then each computer in the network has direct access to a file server. It is sometimes reasonable to assume that this access be available even when one of the file servers goes down. A complete cototal dominating set provides the desired fault tolerance for such cases because each computer has access to at least two file servers and each file server has direct access to one backup server and each backup file server has direct access to at least one backup file server.

\section{Results}

Obviously, a graph with an isolated vertex cannot have a complete cototal dominating set. We ask the natural question regarding the existence of complete cototal dominating sets. 
Theorem 2.1 Every graph with no isolated vertices has a complete cototal dominating set and hence a complete cototal domination number.

Proof. Without loss of generality, let $G=(V, E)$ be connected. Then $V$ itself is a complete cototal dominating set as each vertex is considered to dominate itself, and since $G$ has no isolates, $G$ is nontrivial so each vertex $v$ is adjacent to some other vertex $u$. Thus both $u$ and $v$ dominate $v$ and if $V[G-V]$ has no isolated vertices. Now that we know $G$ has a complete cototal dominating set, we may remove one vertex at a time from $V$ if and only if the remaining subset of $V$ is still a complete cototal dominating set. This will give a minimal complete cototal dominating set. Among all the complete cototal dominating sets, each of the smallest sets has cardinality $\gamma_{C C}(G)$.

Henceforth we consider only graphs with no isolated vertices.

First we calculate complete cototal domination in specific families of graphs.

\section{Theorem 2.2}

(i) For any path $P_{p}, \gamma_{C C}\left(P_{p}\right)=p-2\left\lfloor\frac{p-2}{4}\right\rfloor$

(ii) For any cycle $C_{p}, \gamma_{C C}\left(C_{p}\right)=p-2\left\lfloor\frac{p}{4}\right\rfloor$

(iii) For any star $K_{1, p-1}, \gamma_{C C}\left(K_{1, p-1}\right)=p$

(iv) For any wheel $w_{p}, \gamma_{c C}\left(W_{p}\right)=2$

(v) For any complete graph $K_{p}, \gamma_{C C}\left(K_{p}\right)= \begin{cases}p & \text { if } \mathrm{p}=2,3 \\ 2 & \text { otherwise }\end{cases}$

Next, we state straightforward upper and lower bounds for $\gamma_{C C}(G)$.

Theorem 2.3 Let $G$ be a graph with no isolated vertices. Then $2 \leq \gamma_{C C}(G) \leq p$, and these bounds are sharp. Further, equality of an upper bound holds if and only if every edge of $G$ is incident to a support vertex or $G=C_{3}$.

Proof. Clearly from the definition of $\gamma_{C C}(G)$ and by Theorem 2.2, the result follows.

Now for the equality of the upper bound, if $p=2$ or 3 then the result is obvious. Let us assume that $p \geq 4$. Let $D$ be the complete cototal dominating set of $G$. Then clearly $D$ contains all the pendant vertices and support vertices of a graph $G$. Also no two vertices of $V-D$ are adjacent, which implies that $|D|=p$ is a minimum complete cototal dominating set of $G$.

Suppose every edge of $G$ is not incident to a support vertex or $G \neq C_{3}$, then there exists an edge $u v$ where neither $u$ nor $v$ is a support vertex and $\operatorname{deg}(u) \geq 2$ and $\operatorname{deg}(v) \geq 2$. Let $D=V-\{u, v\}$. Obviously $D$ is a dominating set and $\langle V-D\rangle$ is 
connected. If $\langle D\rangle$ contains no isolated vertices, then $D$ is a complete cototal dominating set of $G$ of cardinality $p-2$, a contradiction.

Next we present the lower bound for $\gamma_{C C}(G)$ in terms of maximum degree.

Theorem 2.4 Let $G=T$ be any nontrivial tree. Then $1+\Delta(G) \leq \gamma_{C C}(G)$. Further equality holds if and only if $G=K_{1, p-1}$.

Proof. Let $G=T$ be any nontrivial tree and let $D$ be a complete cototal dominating set of $T$. Clearly $D$ contains all the pendant vertices and support vertices of a tree. Therefore $\gamma_{C C}(T) \geq 1+\Delta(T)$.

For equality, if $T=K_{1, p-1}$ then by result (iii) of Theorem 2.2, the result follows.

Conversely, let $T$ be a tree with $\gamma_{C C}(T)=1+\Delta(T)$. Let $D$ be a complete cototal dominating set with size $|D|=1+\Delta(T)$ and let $v$ be a maximum degree vertex in $T$. If $v \notin D$, then $|D| \geq 2 \Delta(T)$ which is impossible, so $v \in D$. Now it follows from $|D|=1+\Delta(T)$ that $N(v) \subseteq T$ and any vertex of $N(v)$ is a pendant vertex. Hence $D=K_{1, p-1}$.

Next theorem gives the upper bound for $\gamma_{C C}(G)$ in terms of order and size.

Theorem 2.5 Let $G=(p, q)$ be any nontrivial connected graph. Then $\gamma_{C C}(G) \leq 2 q-p+2$. Further, equality holds if and only if $G=T$ is tree and every edge of a tree is incident with a support vertex.

Proof. Obviously, $\gamma_{C C}(G) \leq p=2(p-1)-p+2$ and since $G$ is connected, $q \geq p-1$. Thus $\gamma_{C C}(G) \leq 2 q-p+2$ if and only if every edge of a tree is incident with a support vertex, then $q=p-1$ and $\gamma_{C C}(G)=p=2 q-p+2$.

Conversely, let $\gamma_{C C}(G)=2 q-p+2$. Then $2 q-p+2 \leq p$, which implies that $q \leq p-1$, so $G$ is a tree with $\gamma_{C C}(G)=p$ and by Theorem 2.3, the result follows.

Next, we give the upper bound for $\gamma_{C C}(G)$ in terms of order and minimum degree.

Theorem 2.6 Let $G$ be any connected graph of order at least four and $2 \leq \delta(G) \leq p-1$.

Then $\gamma_{C C}(G) \leq p-\delta(G)$.

Proof. Let $\delta(G)=x$ be a minimum degree vertex of $G$. We consider the following cases.

Case 1. There exist a vertex $y \in N[x]$ such that $N[y] \neq N[x]$. Let $X=N[x]-\{y\}$ and $D=V-X$. As $|X|=\delta(G)$, each vertex of $X$ has a neighbor in $D$, so $D$ is a dominating set. Also $\langle X\rangle$ contains no isolated vertices. Suppose $u$ is an isolated vertex in $\langle D\rangle$. Then obviously $u \neq y$ and $N(u)=X$. Hence $u$ is a neighbor of $x$. But then 
$\operatorname{deg}(x)>\delta(G)$, a contradiction. Thus we conclude that $D$ is a complete cototal dominating set with cardinality $p-\delta(G)$. Hence $\gamma_{C C}(G) \leq p-\delta(G)$.

Case 2. $N[y]=N[x]$ for every vertex $y \in N(x)$. Then the connectivity of $G$ implies that $G$ is complete graph. But then $\delta(G)=p-1$, a contradiction.

The next theorem relates $\gamma_{C C}(G)$ with maximum number of independent edges $\beta_{1}(G)$ of $G$.

Theorem 2.7 If $G$ has $\delta(G) \geq 2$, then $\gamma_{C C}(G) \leq 2 \beta_{1}(G)$.

Proof. Let $G$ be a graph with $\delta(G) \geq 2$ and $M$ be a maximum independent set of edges in $G$. Let $S \subseteq M$ be the total dominating set of $G$. Since each $v \in V-S$ must have at least one neighbor in $V-S$. So $S$ is a complete cototal dominating set of $G$. Hence $\gamma_{C C}(G) \leq 2 \beta_{1}(G)$.

Theorem A[1] For any graph $G$ without isolated vertices, $\gamma(G) \geq\left\lceil\frac{p}{\Delta(G)+1}\right\rceil$.

Relates the domination number to the maximum degree. We establish a similar result for $\gamma_{C C}(G)$.

Theorem 2.8 For any graph $G$, without isolated vertices, $\frac{2 p}{\Delta(G)+1} \leq \gamma_{C C}(G)$

Proof. Let $G$ have no isolated vertices and let $S$ be a $\gamma_{C C}$ - set for $G$. Let $t$ denote the number of edges in $G$ having one vertex in $S$ and the other vertex in $V-S$. Since $\Delta(G) \geq \operatorname{deg}(v)$ for all $v \in S$ and each vertex in $S$ is adjacent to at least one member of $S$, we have, $t \leq(\Delta(G)-1)|S|=(\Delta(G)-1) \gamma_{C C}(G)$. Also each vertex in $V-S$ is adjacent to at least one vertex of $V-S$, we have, $t \geq 2|V-S|=2\left[P-\gamma_{C C}(G)\right]$

Hence, $2\left(p-\gamma_{S S}(G)\right) \leq \Delta(G) \gamma_{C C}(G)-\gamma_{C C}(G)$.

This reduces to the bound of the theorem.

Theorem 2.9 For any tree $T \neq K_{1, p-1}$ with $p \geq 6$, then $\gamma_{C C}(T) \geq\left\lceil\frac{2 p}{3}\right\rceil$.

In the next theorem we characterize the tree for which $\gamma_{C C}(T)=\frac{2 p}{3}$.

We define the following operations on trees which achive the lower bound of the above theorem 2.9.

Operation 1 Attach $P_{3}$ to a vertex $v$, where $v$ is neither a pendant vertex nor a support vertex and $N(v)$ has a support vertex of degree two in $T$.

Operation 2 Attach $P_{1}$ to a vertex $v$, where $v$ is either a pendant vertex or a support vertex of $T$.

Operation 3 Attach $P_{2}$ to any vertex of $T$.

Let, $X_{0}=\left\{T / \mathrm{T}\right.$ is a tree obtained from $\mathrm{P}_{6}$ by finite type of operations of type 1$\}$

$X_{1}=\left\{T /\right.$ is obtained from a tree T' $T \in X_{0}$ by applying operation 2 to $\left.\mathrm{T}^{\prime}\right\}$

$X_{2}=\{T / \mathrm{T}$ is a tree which can be obtained from a tree $T \in X$ by applying operation 3$\}$ 
Let, $\tau_{n}=\left\{T / T\right.$ is a tree of order p such that $\left.\gamma_{C C}(T)=\frac{2 p}{3}\right\}$

Proposition 1 If $T \in X_{0}$ has order $p \geq 6$, then $D(T)=P(T) \cup S(T)$ is a complete cototal dominating set of cardinality $\frac{2 p}{3}$. Where $\mathrm{D}(\mathrm{T})$ is the union of set of all pendant vertices and the support vertices of a tree $\mathrm{T}$.

Proof. We use induction on $k$, the number of steps required to construct $T$. If $k=0$, then $T \cong P_{6}$, and $D(T)$ is a complete cototal dominating set of cardinality 4, i.e. $\frac{2 p}{3}=\frac{2 * 6}{3}=4$. Suppose $T$ can be constructed from $P_{6}$ by a sequence of $k \geq 1$ operations of type 1 , and suppose that $T^{\prime}$ is a tree which can be obtained from $P_{6}$ by $k-1$ operations of type 1 , then let $D\left(T^{\prime}\right)$ is a complete cototal dominating set of cardinality $\frac{2 p\left(T^{\prime}\right)}{3}$. As $T \in X_{0}$, $T$ can be obtained from $T^{\prime}$ by applying operation type 1 . By induction assumption $D\left(T^{\prime}\right)=P\left(T^{\prime}\right) \cup S\left(T^{\prime}\right)$ is a complete cototal dominating set of cardinality $\frac{2 p\left(T^{\prime}\right)}{3}$. Therefore, $D\left(T^{\prime}\right) \cup\left\{v_{1}, v_{2}\right\}=D(T)$ is a complete cototal dominating set of cardinality $\frac{2 p(T)}{3}$.

Proposition 2 Let $T \in \tau$ and $v$ is a pendant vertex of $T$. If $D-\{v\}$ is a complete cototal dominating set of $T-v$, then the number of vertices in a tree is not a multiple of 3 and $T-v \in \tau$.

Proof. Suppose $V$ is a pendant vertex of a tree $T$, and $D-\{v\}$ is a complete cototal dominating set of $T-\{v\}$. Note that $\left\lceil\frac{2(p-1)}{3}\right\rceil \leq \gamma_{C C}(T-v) \leq\left\lceil\frac{2 p}{3}\right\rceil-1$.

If the number of vertices in a tree $T$ is a multiple of three, i.e. $p=3 k$ for some positive integer $k \geq 1$, then we have $\left\lceil\frac{2(3 k-1)}{3}\right\rceil \leq\left\lceil\frac{2(3 k)}{3}\right\rceil-1$, and so, $2 k \leq 2 k-1$, which is impossible. Thus $p=3 k+1$ or $p=3 k+2$ for some positive integer $k \geq 1$.

If $p=3 k+1$, then $\left\lceil\frac{2(3 k)}{3}\right\rceil \leq \gamma_{C C}(T-v) \leq\left\lceil\frac{2(3 k+1)}{3}\right\rceil-1$, which implies that, $2 k \leq \gamma_{C C}(T-v) \leq 2 k$ and so $T-v \in \tau$.

If $p=3 k+2$, then $\left\lceil\frac{2(3 k+1)}{3}\right\rceil \leq \gamma_{C C}(T-v) \leq\left\lceil\frac{2(3 k+2)}{3}\right\rceil-1$, which implies $2 k+1 \leq \gamma_{C C}(T-v) \leq 2 k+1$ and so $T-v \in \tau$.

Proposition 3 Let $T \in \tau$ and suppose $v_{1}$ and $v_{2}$ are either both pendant vertices of $T$ or $v_{1}$ is degree two support vertex of $T$ adjacent to a pendant vertex $v_{2}$. If $D-\left\{v_{1}, v_{2}\right\}$ is a 
complete cototal dominating set of $T-v_{1}-v_{2}$, then $T-v_{1}-v_{2} \in \tau$ and the number of vertices in a tree minus two is a multiple of three.

Proof. Suppose $v_{1}$ and $v_{2}$ are either pendant vertices of a tree $T$ or $v_{1}$ is a degree two support vertex of $T$ adjacent to a pendant vertex $v_{2}$ and that $D-\left\{v_{1}, v_{2}\right\}$ is a complete cototal dominating set of $T-v_{1}-v_{2}$. Note that $\left\lceil\frac{2(p-2)}{3}\right\rceil \leq \gamma_{C C}\left(T-v_{1}-v_{2}\right) \leq\left\lceil\frac{2 p}{3}\right\rceil-2$.

Suppose either $p=3 k$ or $p=3 k+1$ for some positive integer $k \geq 1$.

If $p=3 k$, then $\left\lceil\frac{2(3 k-2)}{3}\right\rceil \leq\left\lceil\frac{2(3 k)}{3}\right\rceil-2$, and so $2 k-1 \leq 2 k-2$, which is impossible.

If $p=3 k+1$, then $\left\lceil\frac{2(3 k-1)}{3}\right\rceil \leq\left\lceil\frac{2(3 k+1)}{3}\right\rceil-2$, and so, $2 k \leq 2 k-1$, which is impossible.

Thus, $p=3 k+2$. Hence $p=3 k+2$ for some positive integer $k$, and so $\left\lceil\frac{2(3 k)}{3}\right\rceil \leq\left\lceil\frac{2(3 k+2)}{3}\right\rceil-2$.

Hence, $2 k \leq \gamma_{C C}\left(T-v_{1}-v_{2}\right) \leq 2 k$ and so $T-v_{1}-v_{2} \in \tau$.

Now we are ready to prove the following theorem.

Theorem 2.10 For any tree $T \neq K_{1, p-1}$ with $p \geq 6, \tau=\cup_{i=0}^{2} X_{i}$.

Proof. Suppose $T \in X_{0}$. If $T \cong P_{6}$, then $\gamma_{C C}(T)=4=\left\lceil\frac{2 p(T)}{3}\right\rceil$. If $T$ is not $P_{6}$ then by Proposition 1, $\gamma_{C C}(T)=\left\lceil\frac{2 p(T)}{3}\right\rceil$ and so $T \in \tau$.

Suppose $T \in X_{1}$. Then $p=p(T)=3 k+1$ for some positive integer $k$, and $T$ can be constructed from $T^{\prime} \in X_{0}$ by applying operation 2 to $T^{\prime}$. Let $u$ be the $P_{1}$ attached. By Proposition $1, D\left(T^{\prime}\right)$ is a complete cototal dominating set of cardinality $2 k$. But then $D \cup\{u\}$ is a complete cototal dominating set of $T$ of cardinality $2 k+1$. Therefore, $\gamma_{C C}(T) \geq\left\lceil\frac{2(3 k+1)}{3}\right\rceil=2 k+1$ and so $\gamma_{C C}(T)=\left\lceil\frac{2 p}{3}\right\rceil$. Hence $T \in \tau$.

Suppose $T \in X_{2}$, then $p=p(T)=3 k+2$ for some positive integer $k$, and $T$ can be constructed from $T^{\prime} \in X_{0}$ by applying operation 3. Let $u$ and $u^{\prime}$ be the added vertices. By Proposition $1, D\left(T^{\prime}\right)$ is a complete cototal dominating set of cardinality $2 k$. But then $D \cup\left\{u, u^{\prime}\right\}$ is a complete cototal dominating set of $T$ of cardinality $2 k+2$. Therefore $\gamma_{C C}(T) \geq\left\lceil\frac{2(3 k+2)}{3}\right\rceil=2 k+2$, and so $\gamma_{C C}(T)=\left\lceil\frac{2 p}{3}\right\rceil$. Hence $T \in \tau$. 
We now show that $\tau \subseteq \cup_{i=0}^{2} X_{i}$. To prove this we apply induction on the number of vertices $p$ of the tree $T \in \tau$. If $p=6$, then $T \cong P_{6} \in X_{0}$. Suppose $T \in \tau$ has order $p=p(T) \geq 1$ and assume that $T^{\prime}$ is a tree with $6 \leq p\left(T^{\prime}\right)<p$ and $T^{\prime} \in \tau$. Then $T^{\prime} \in \cup_{i=0}^{2} X_{i}$. Let $D$ be any complete cototal dominating set. Then each vertex in $V-D$ is adjacent to at least one vertex in $D$. Furthermore, every vertex in $D$ is either a degree two support vertex adjacent to exactly one vertex in $V-D$ or a pendant vertex adjacent to degree two support vertex that is adjacent to exactly one vertex in $V-D$. Hence the number of vertices in a tree is the multiples of three and $p=3 k$ for some positive integer $k \geq 2$. Let $v$ be the end point of the diametrical path $v=v_{1}, v_{2}, \cdots, v_{i}$ in $\langle V-D\rangle$. As $T$ contains no cycles, $v$ is a pendant vertex of $\langle V-D\rangle$. Thus $\operatorname{deg}(v)=2$ and $|V-D| \geq 2$, and so $v_{2}$ is adjacent to a degree two support vertex. If $|V-D|=2$, then $T \cong P_{6} \in X_{0}$. We therefore assume that $|V-D| \geq 3$. Note that $v_{2}$ is neither a pendant vertex nor a support vertex of $T^{\prime}=T-v_{1}-u_{0}-u_{1}$. Moreover, $D-\left\{u_{0}, u_{1}\right\}$ is a complete cototal dominating set of $T^{\prime}$ and so $\left\lceil\frac{2(3 k-3)}{3}\right\rceil \leq \gamma_{C C}\left(T^{\prime}\right) \leq\left\lceil\frac{2(3 k)}{3}\right\rceil-2$, which implies that $2 k-2 \leq \gamma_{C C}\left(T^{\prime}\right) \leq 2 k-2$ and so $T^{\prime} \in \tau$. By our induction assumption $T^{\prime} \in X_{0}$. Hence $T \in X_{0}$.

Next, we have the following characterization of those graphs for which the complete cototal domination number is equal to the total domination number.

Theorem 2.11 For any graph $G, \gamma_{C C}(G)=\gamma_{t}(G)$ if and only if $G$ has a total dominating set $D$ such that $\langle V-D\rangle$ has no isolated vertices.

In the next theorem we prove the relation between connected domination number and complete cototal domination number.

Theorem 2.12 If $\Delta(G)=p-1$, then $\gamma_{C C}(G) \geq 1+\gamma_{C}(G)$.

Proof. If $\Delta(G)=p-1$. Then clearly, $\gamma_{C}(G)=1$. By the definition of complete cototal domination and by Theorem 2.3, $\gamma_{C C}(G) \geq 2$. Therefore, clearly $\gamma_{C C}(G) \geq \gamma_{C}(G)+1$.

Next theorem gives the relation between cototal domination number and the complete cototal domination number.

Theorem 2.13 For any graph $G$, a cototal dominating set $D$ is said to be a complete cototal dominating set of $G$ if $\langle D\rangle$ has no isolated vertices.

\section{Nordhaus-Gaddum type results}

Theorem 2.14 Let $G$ be any nontrivial connected graph. If both $G$ and $\bar{G}$ has no isolated vertices, then 
(i) $\gamma_{C C}(G)+\gamma_{C C}(\bar{G}) \leq 2 p$

(ii) $\gamma_{C C}(G) \cdot \gamma_{C C}(\bar{G}) \leq P^{2}$

Further, equality holds for $G=P_{4}$ or $C_{5}$.

Finally we conclude this paper for exploring the following two open problems for further research on this topic: Open problem 1 - to characterize the graphs for which $\gamma_{C C}(G)=\gamma_{C l}(G)$; Open problem 2 - to characterize the graphs for which $\gamma_{C C}(G)=1+\gamma_{C}(G)$.

\section{Conclusion}

In this paper, we introduce the new parameter $\gamma_{c c}(G)$ of $G$, in the field of domination theory in graphs. We obtained many bounds for $\gamma_{c c}(G)$. We also characterized the extremal trees which have $\gamma_{C C}(T)=\frac{2 p}{3}$. Finally we compare $\gamma_{c c}$ with $\gamma_{c}(G), \gamma_{t}(G)$ and $\gamma_{c l}(G)$, respectively and concluded with exploring the two open problems related to this parameter.

\section{Acknowledgement}

B. Basavanagoud acknowledges the support of UGC-SAP DRS-II New Delhi, India for the peiod 2010-2015, and Sunilkumar. M. Hosamani acknowledges the support of the University Grants Commission, New Delhi, India (No. F.4-3/2006(BSR)/7-101/2007(BSR), September 2009).

\section{References}

1. F. Harary, Graph Theory (Addison-Wesley, Reading, Mass, 1969).

2. T. W. Haynes, S. T. Hedetniemi, and P. J.Slater (Fundamentals of domination in graphs, Marcel Dekker, Inc, New York , 1998).

3. E. Sampathkumar and H. B.Walikar, J. Math. Phys. Sci. 13, 607 (1979).

4. E. J. Cockayne, R. M. Dawes, and S. T. Hedetniemi, Networks 10, 211 (1980). http://dx.doi.org/10.1002/net.3230100304

5. V. R. Kulli, B. Janakiram and R. R.Iyer, Discrete Mathematical Sciences and Cryptography 2, 179 (1999).

6. E. J. Cockayne and S. T. Hedetniemi, Networks 7, 247 (1977). http://dx.doi.org/10.1002/net.3230070305 